Discrete Comput Geom 30:467-487 (2003)

DOI: $10.1007 / \mathrm{s} 00454-003-2846-4$

\title{
Tilings of the Sphere with Isosceles Triangles*
}

\author{
Robert J. MacG. Dawson \\ Department of Mathematics and Computing Science, St. Mary's University, \\ Halifax, Nova Scotia, Canada B3H 3C3 \\ rdawson@smu.ca
}

\begin{abstract}
The spherical triangles which tile the sphere in an edge-to-edge fashion have been known for some time. However, if we relax the requirement that the triangles must meet edge-to-edge, other tilings are possible. This paper begins the classification of these tilings by characterizing all isosceles triangles that tile the sphere. One infinite family and three sporadic tiles that tile only edge-to-edge are exhibited.
\end{abstract}

\section{Introduction}

A homohedral tiling is one in which all tiles are congruent. (Grünbaum and Shepherd have "monohedral" [5], but this suggests more that the tiling has a single tile rather than a single prototile.) In 1923 Sommerville [11] classified the edge-to-edge homohedral tilings of the sphere with isosceles triangles, and those with scalene triangles in which the angles meeting at any one vertex are congruent. Davies [3] completed the classification of edge-to-edge homohedral tilings by triangles in 1965 (apparently without knowledge of Somerville's work), allowing any combination of angles at a vertex. Davies' paper left some details to the reader; these have been supplied recently in the very detailed and complete work of Ueno and Agaoka [12]. In another related paper [1], Azavedo Breda has classified edge-to-edge triangulations of the sphere with certain conditions on the patterns of angles at each vertex.

If the restriction to edge-to-edge tilings is removed, a number of new tilings may be found. Some of these (see below) use tiles that also tile edge-to-edge; there are also some triangles that tile only in a non-edge-to-edge fashion. This paper is a first step towards the enumeration of these triangles, dealing with the isosceles case. The equilateral case will be dealt with briefly at the beginning of the third section; elsewhere, we always assume "isosceles" to mean "isosceles and not equilateral."

* This research was supported by a grant from NSERC. 
In Section 3 we examine the isosceles triangles that tile edge-to-edge, to determine which also permit a non-edge-to-edge tiling; in Sections 4 and 5 we enumerate the triangles which permit only a non-edge-to-edge tiling. We do not always determine in how many ways such a triangle tiles the sphere; however, we shall determine in each case whether it tiles in a non-edge-to-edge fashion.

\section{Definitions, Notation, and Preliminaries}

Throughout this paper we represent the measure of the large angle of the triangle by $\alpha$ and that of the small angle by $\beta$. We call any isosceles triangle with two large angles tall, and one with two small angles short. It is convenient to treat these two cases independently.

A maximal arc of a great circle that is contained in the union of the edges, and is longer than any edge contained in it, is called an extended edge. A triangle with angles $\left(\theta, 90^{\circ}, 90^{\circ}\right)$ is called a right semilune, and one with angles $\left(\theta, \theta, 180^{\circ}-\theta\right)$ is called an isosceles semilune. (It should be noted that this is a slight abuse of notation, as the right semilunes are also isosceles, but with the equal edges oriented differently with respect to the lune.) In either case, the angle $\theta$ is called the lune angle.

We call the lengths of the edges opposite the large and small angles $L$ and $S$ ("long" and "short"), respectively. It is highly plausible that, except for a few cases such as equilateral triangles and right semilunes, $L / S$ is always irrational when the angles (in degrees) are rational, from which it would follow that the numbers of long and short edges on one side of an extended edge must equal those on the other side. However, this brings in deep and difficult questions of transcendence theory that we should like to avoid. We will occasionally need special cases of this hypothesis that can be verified by direct calculation.

We make a great deal of use of the vertex equations satisfied by a given triangle. These are the equations $a \alpha+b \beta=360^{\circ}$ satisfied by the angles of a triangle, regardless of whether any vertex with these angles actually appears in a tiling. For brevity, such an equation is represented by the vertex vector $(a, b)$. A non-edge-to-edge tiling must also have split vertices: vertices surrounded by two or more triangle angles and a straight angle, on the relative interior of an edge. The angles at such a vertex satisfy $a \alpha+b \beta=180^{\circ}$; we call this a split equation, and abbreviate it as $(2 a, 2 b) / 2$. For instance, a $\left(90^{\circ}, 60^{\circ}, 60^{\circ}\right)$ triangle has (among others) the vertex vectors $(2,3)$ and $(4,0)$, and the split vector $(4,0) / 2$. The following result is trivial, and is stated for later reference:

Lemma 1 (Angle Comparison Lemma). If a triangle has the vertex vector $(a, b)$, then it cannot have a vertex vector $(c, b)$ with $c \neq a$, a vertex vector $(a, d)$ with $d \neq b$, or $a$ vertex vector $(c, d)$ with $(c+d-a-b)(c-a)>0$.

The excess, $\varepsilon$, of a spherical triangle is defined to be the sum of the angles minus $180^{\circ}$; in our notation, $2 \alpha+\beta-180^{\circ}$ for a tall triangle and $\alpha+2 \beta-180^{\circ}$ for a short triangle. Recall that $\varepsilon>0$ for any spherical triangle, and that $N=720^{\circ} / \varepsilon$, where $N$ is (here and henceforth) the number of triangles in the tiling. The following result simplifies testing sphericity. 
Lemma 2 (Sphericity Lemma). If a spherical triangle has two vertex vectors $(a, b)$ and $(c, d)$ with $a>c$, then

$$
(a-4)(d-2)<(c-4)(b-2)
$$

if the triangle is tall, and

$$
(a-2)(d-4)<(c-2)(b-4)
$$

if the triangle is short.

Proof. If $a>c$, we have $b<d$, and $a d-b c>0$. By Cramer's rule we have

$$
\begin{aligned}
& \alpha=360^{\circ}(d-b) /(a d-b c), \\
& \beta=360^{\circ}(a-c) /(a d-b c),
\end{aligned}
$$

whence (in the tall case) $4(d-b)+2(a-c)>(a d-b c)$. Rearranging, we get $4 d+2 a-a d>4 b+2 c-b c$; adding 8 to each side and factoring, the result follows. The short case works in the same way.

Another elementary result which is occasionally useful is obtained by observing that if a triangle has angles $(\alpha, \beta, \gamma)$ (here, in no particular order), and we extend the sides opposite $\alpha$ and $\beta$ until they intersect again, they create a second triangle with sides $\left(180^{\circ}-\alpha, 180^{\circ}-\beta, \gamma\right)$; and the excess of that triangle must be positive as well:

Lemma 3. For any spherical triangle, the sum of any two angles is less than $180^{\circ}$ plus the third.

\section{New Tilings from Old Tiles}

The equilateral triangles that tile the sphere are those with angles $\left(180^{\circ}, 180^{\circ}, 180^{\circ}\right)$ (degenerate), $\left(120^{\circ}, 120^{\circ}, 120^{\circ}\right),\left(90^{\circ}, 90^{\circ}, 90^{\circ}\right)$, and $\left(72^{\circ}, 72^{\circ}, 72^{\circ}\right)$. The edge-to-edge tilings of the first and third type of triangle have extended edges that are great circles. Any such tiling can be converted into any of a continuum of non-edge-to-edge tilings by rotating one hemisphere relative to the other. (We call tilings obtained in this way from edge-to-edge tilings twisted.) These are the only non-edge-to-edge tilings with equilateral triangles; the other two are easily seen only to tile in an edge-to-edge fashion, as their angles do not divide $180^{\circ}$.

The nonequilateral isosceles triangles that tile edge-to-edge are given in Table 1. Their edge-to-edge tilings are catalogued in [3]; some of them also permit non-edge-to-edge tilings. The triangles of the form $\left(\theta, 180^{\circ}-\theta / 2,180^{\circ}-\theta / 2\right)$ for $90^{\circ}<\theta<180^{\circ}$ have $\varepsilon=180^{\circ}$ and only four of them cover the sphere. They only tile edge-to-edge, although in the degenerate cases $\theta=90^{\circ}, 180^{\circ}$, the tile is a $90^{\circ}$ lune and there are twisted tilings.

Proposition 1. The triangles $\left(120^{\circ}, 36^{\circ}, 36^{\circ}\right),\left(72^{\circ}, 60^{\circ}, 60^{\circ}\right)$ tile only edge-to-edge and the triangle $\left(120^{\circ}, 45^{\circ}, 45^{\circ}\right)$ tiles only edge-to-edge or in twisted tilings derived from the edge-to-edge tiling. 
Table 1. Isosceles triangles that tile the sphere.*

\begin{tabular}{lcccc}
\hline \multicolumn{1}{c}{ Triangle } & $N$ & ETE & Non-ETE & Figure \\
\hline$\left(\theta, 180^{\circ}-\theta / 2,180^{\circ}-\theta / 2\right)$ & 4 & 1 & 0 & \\
$\left(120^{\circ}, 60^{\circ}, 60^{\circ}\right)$ & 12 & 7 & $\mathrm{c}$ & \\
$\left(120^{\circ}, 45^{\circ}, 45^{\circ}\right)$ & 24 & 1 & $\mathrm{c}$ & \\
$\left(120^{\circ}, 36^{\circ}, 36^{\circ}\right)$ & 60 & 1 & 0 & \\
$\left(90^{\circ}, 60^{\circ}, 60^{\circ}\right)$ & 24 & 1 & $\mathrm{c}$ & \\
$\left(72^{\circ}, 60^{\circ}, 60^{\circ}\right)$ & 60 & 1 & 0 & \\
$\left(360^{\circ} / n, 90^{\circ}, 90^{\circ}\right)$ & $2 n$ & 2 & & \\
$n$ even & $2 n$ & 1 & $\mathrm{c}$ & \\
$n$ odd & $4 n$ & 1 & 0 & \\
$\left(360^{\circ} / n,\left(90^{-} 90 / n\right)^{\circ},(90-90 / n)^{\circ}\right)$ & $4 n$ & 2 & 0 & 1 \\
$n$ even & & & & \\
$n$ odd & $2 n$ & 1 & $\mathrm{c}$ & 8 \\
$\left((180-360 / n)^{\circ},(360 / n)^{\circ},(360 / n)^{\circ}\right)$ & $2 n$ & 2 & $\mathrm{c}$ & \\
$n \equiv 0 \bmod 4$ & $2 n$ & 0 & many & \\
$n \equiv 2 \bmod 4$ & 36 & 0 & 3 & 14 \\
$n$ odd & 18 & 0 & 1 & 16 \\
$\left(80^{\circ}, 60^{\circ}, 60^{\circ}\right)$ & 8 & 0 & 1 & 20 \\
$\left(100^{\circ}, 60^{\circ}, 60^{\circ}\right)$ & & & \\
$\left(150^{\circ}, 60^{\circ}, 60^{\circ}\right)$ & & & & \\
\hline
\end{tabular}

*Numbers of ETE tilings are taken from [3]. The entry "c" indicates a continuous family of tilings.

Proof. Each triangle has only vertex vectors of the forms $(a, 0)$ and $(0, b)$, in which the single vertex appears an odd number of times. It follows that every triangle in a tiling with one of these must be part of a supertile of three or five triangles. In the first two cases the supertile (and thus the original tile) tiles only edge-to-edge; in the third case the supertile is a $\left(90^{\circ}, 90^{\circ}, 90^{\circ}\right)$ triangle that, as observed above, permits a twisted tiling.

The $\left(120^{\circ}, 60^{\circ}, 60^{\circ}\right)$ triangle can tile in several ways. Two of them make up a $60^{\circ}$ lune, or a regular quadrilateral with $120^{\circ}$ angles (the face of a spherical cube). Both of these tile the sphere, as does a combination of two quadrilaterals and four lunes; and, in either case, the symmetry group of the union is larger than that of the configuration, and permits multiple tilings. Many of these are non-edge-to-edge.

The $\left(120^{\circ}, 60^{\circ}, 60^{\circ}\right)$ triangle is the $n=3$ case of the infinite family of triangles of the form $\left(360^{\circ} / n,(90-90 / n)^{\circ},(90-90 / n)^{\circ}\right.$ ) (see the left of Fig. 1; when $n=5$ we get the icosahedron). All these triangles tile the sphere edge-to-edge, with $n$ small angles (or large angles for $n=3,4$ ) meeting at each pole, and an equatorial belt of triangles pointing alternately up and down. As shown by Sommerville and Davies, if $n$ is odd there is a second tiling (Fig. 1, right) in which half the tiles are rotated relative to the rest. If $n=5$ the action of the rotation is trivial.

Proposition 2. A triangle of the form $\left(360^{\circ} / n,(90-90 / n)^{\circ},(90-90 / n)^{\circ}\right), n>3$, tiles only edge-to-edge. 


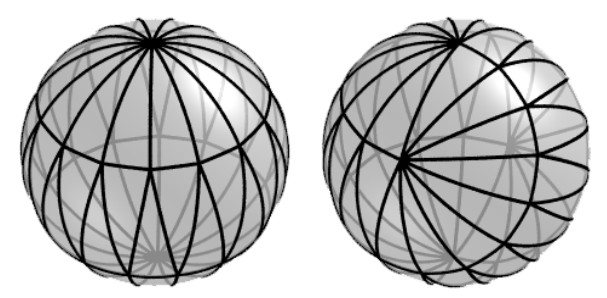

Fig. 1. The only tilings with the $\left(360^{\circ} / n,(90-90 / n)^{\circ},(90-90 / n)^{\circ}\right)$ triangle.

Proof. First, we consider the special case $n=4$. This triangle is short and has only the vertex vectors $(4,0)$ and $(1,4)$. It tiles only edge-to-edge; this follows from the following lemma, which we will also need elsewhere.

Lemma 4. A short triangle whose only split vertex vector is $(4,0) / 2$ has no tilings that are not edge-to-edge.

Proof. The only possible split vertex configuration would have two $90^{\circ}$ angles meeting (Fig. 2).

The resulting extended edge would contain two short edges $\overline{A B}$, with a small angle at either end; as no split vertex contains a small angle, the extended edge would be precisely $\overline{B B}$. This can only be filled on the other side by two other short edges, so that $A$ is not a split vertex.

The equilateral case $n=5$ is easily ruled out as having no split vertices. For $n>5$ the triangle is tall and has the vertex vectors $(4,1)$ and $(0, n)$. If $n \equiv 1 \bmod 4$, the triangle has precisely the vertex vectors $(4,1),(2,(n+1) / 2)$, and $(0, n)$. As none of these split, a non-edge-to-edge tiling is immediately ruled out.

If $n$ is even, the triangle has only the vertex vectors $(4,1)$ and $(0, n)$. The two $\alpha$ corners of any triangle must both be at $(4,1)$ vertices. As there are no split vectors using $\alpha$, long edges must be matched with long edges; this forces a $(4,1)$ vertex to be as at $A$ in Fig. 3 . The neighborhoods of the vertices $B B$ are now in turn forced, and the unique tiling, which is edge-to-edge ("Isosceles case IIb" on page 49 of [3]), results by induction.

If, on the other hand, $n \equiv 3 \bmod 4$, there is a split vector of the form $(2,(n+1) / 2) / 2$. We will show that no split vertex of this type can exist. Firstly, the large angle cannot be between two small angles (Fig. 4(a)). For then the short side of that triangle would

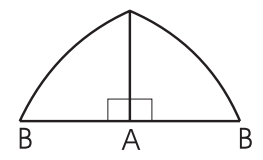

Fig. 2. The neighborhood of a hypothetical split vertex. 


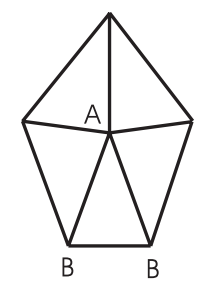

Fig. 3. The $(4,1)$ vertex figure.

be paired with a long side, and triangles 1,1 would be forced at $B$. One of these would overhang at $C$, forcing triangle 2 ; and 2 in turn would overhang at $D$, forcing 3 . However, 3 would overhang at $X$, requiring a split vertex with two large angles, which does not exist.

Next, the large angle of the split vertex must have its long edge paired with the (long) edge of the adjacent small corner, not with the extended edge (Fig. 4(b)). Otherwise triangles 1 and 2 are forced, and again an impossible split vertex is required. Now, the edge $\overline{A B}$ of Fig. 4(c) cannot extend past $B$; otherwise triangle 1 is forced and again an illegal split vertex is required.

Finally, the edge $A^{\prime} B$ cannot extend past $A^{\prime}$ (Fig. 4(d)); otherwise there is a split vertex at $A^{\prime}$ forcing triangles $1 \cdots 1$. As just shown, the extended edge $\overline{B^{\prime} B}$ cannot continue past $B^{\prime}$; so it has length $2 L+S$. This must be paired with three edges of the same lengths on the other side of $\overline{B^{\prime} B}$; and as we have seen, the short edge must be in the middle. Thus the vertices $A^{\prime}, A$ are not split. We thus rule out any configuration for the neighborhood of a hypothetical split vertex except for that of Fig. 5.

Now, vertex $B$ of Fig. 6 must have a neighborhood as shown. In the case $n \geq 11$, this follows immediately from the presence of two $\alpha$ corners at each adjacent vertex (Fig. 6(a)). For $n=7$, triangle 1 is forced in that way, but the next vertex has a $\beta$ corner. However, if the configuration were not as in Fig. 6(a), triangles 2 and 3 of Fig. 6(b) would be forced; and we would then have a split vertex at $D$ with the $\alpha$ angle oriented incorrectly (recall Fig. 4(b)).

We now consider what angles might fill the gap at $A$ in Fig. 6(a), along the edge $\overline{A C}$. If (triangle 1 in Fig. 7(a)) it were an $\alpha$ with the short edge on $\overline{A C}$, triangles 2 and 3 would be forced, and there would be an impossible configuration at $X$.
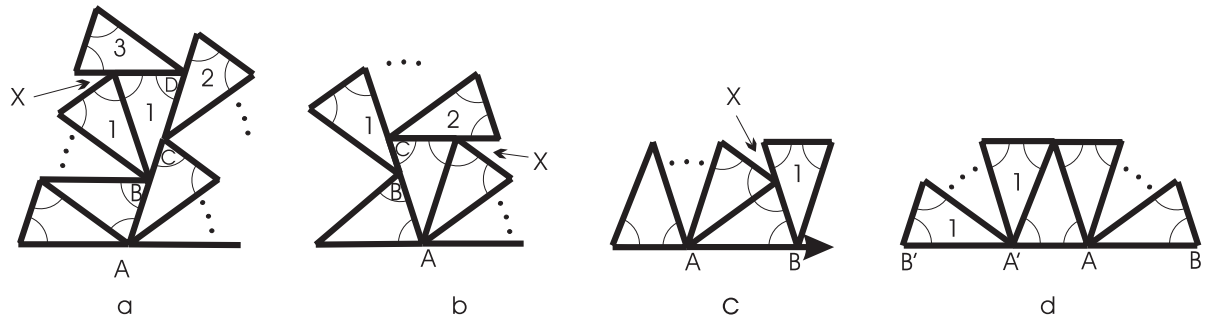

Fig. 4. Some impossible configurations for split vertices. 


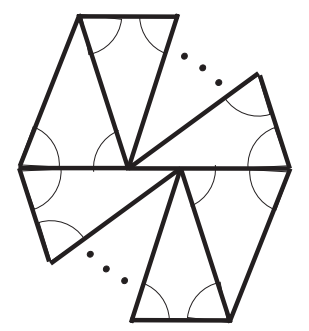

Fig. 5. The neighborhood of a hypothetical split vertex.

If (triangle $1^{\prime}$ in Fig. 7(b),(c)) it were a $\beta$ corner, the remaining gap at $A$ could be filled by an $\alpha$ corner in one of two ways. In Fig. 7(b) the short edge of triangle 2 is against $\overrightarrow{A F}$; triangle 3 is forced. The gap at vertex $F$ needs to be filled with $\beta$ corners, but this cannot be done due to the reflex angle at $C$. If, on the other hand, we have a triangle $2^{\prime}$ with a long edge against $\overline{G A}$ (Fig. 7(c)), it forces a triangle 3 as shown; but we have seen that a split vertex cannot have the configuration at $G$. Finally, we might have a triangle such as $1^{\prime \prime}$ in Fig. 7(d). This forces triangles 2 and 3, and the gap at vertex $H$ cannot be filled.

We conclude that the split vertex $(2,(n+1) / 2) / 2$ cannot occur in a tiling of the entire sphere with such a triangle. Thus, this triangle cannot tile in a non-edge-to-edge fashion.

Triangles of the forms $\left(90^{\circ}, 60^{\circ}, 60^{\circ}\right),\left(360^{\circ} / n, 90^{\circ}, 90^{\circ}\right)$, and $\left(180^{\circ} / n, 180^{\circ}-\right.$ $\left.180^{\circ} / n, 180^{\circ}-180^{\circ} / n\right)$ also have edge-to-edge tilings of the sphere in which great circles are contained in the union of the edges. As above, these yield a continuum of twisted tilings.

Triangles of the form $\left(180^{\circ} / n,(180-180 / n)^{\circ},(180-180 / n)^{\circ}\right)$ tile the sphere as shown in Fig. 8 , with $2 n$ lunes each divided into two triangles. Any of these lunes can be replaced by its mirror image to yield a non-edge-to-edge packing. We may call these tilings flippant. Enumerating them is equivalent to enumerating the labelings of each of the $2 n$ lunes with one of the marks $\backslash$ or $/$. This is done most easily using Burnside's theorem. The number is always approximately $2^{2 n-2} / n$; details are omitted (but see Proposition 11 for a similar calculation).
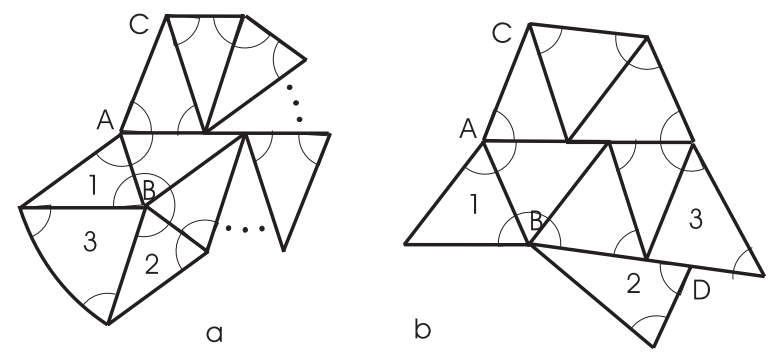

Fig. 6. A forced neighborhood configuration. 

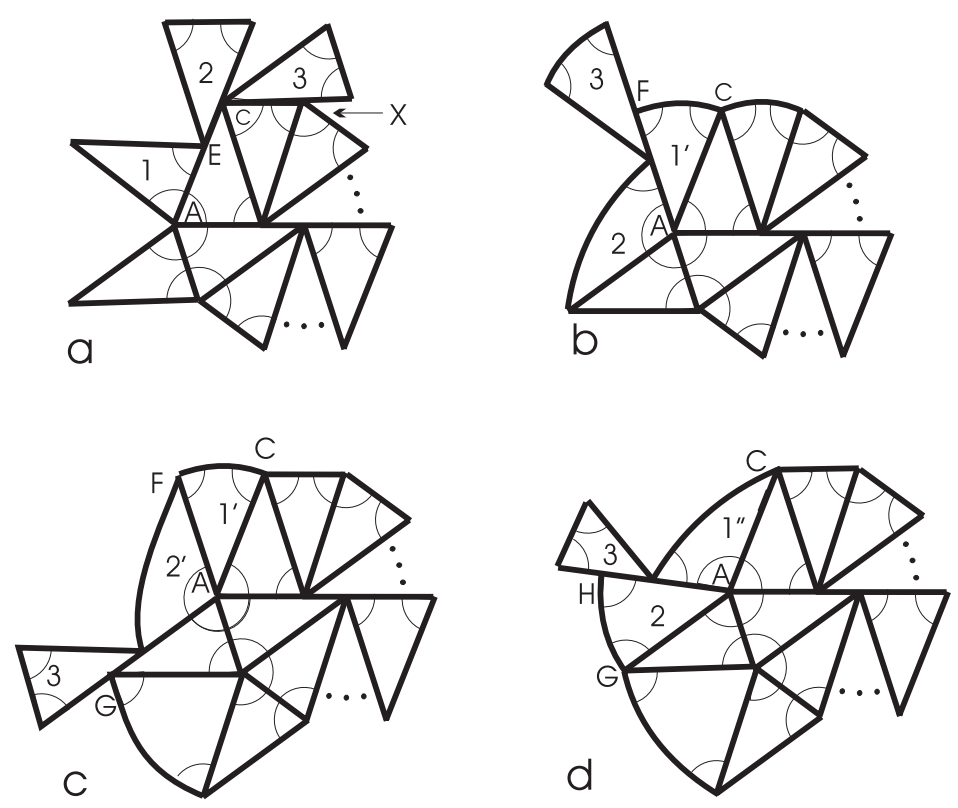

Fig. 7. A gap that cannot be filled.

Triangles of the form $\left(360^{\circ} / n, 90^{\circ}, 90^{\circ}\right)$ also tile in pairs that make up lunes, but the lunes are invariant under reflection. However, if $n$ is even, the triangle $\left(360^{\circ} / n, 90^{\circ}, 90^{\circ}\right)$ tiles a lune with angle $90^{\circ}$, in a "fan" centered on the midpoint of one side. Three of the four essentially distinct ways of arranging these fans are non-edge-to-edge (Fig. 9, left).

The edge-to-edge tiling by the triangle $\left(90^{\circ}, 60^{\circ}, 60^{\circ}\right)$ divides into six asymmetric lunes of four triangles each; each of these can be independently flipped.

Finally, if $n$ is divisible by 4 , the triangle $\left(360^{\circ} / n, 90^{\circ}, 90^{\circ}\right)$ tiles the triangle $\left(90^{\circ}, 90^{\circ}\right.$, $90^{\circ}$ ) in a fan. There are 252 essentially different ways of orienting these fans within a regular "octahedral" tiling (Fig. 9, right), of which two are edge-to-edge . All of these tilings have twisted variants as well.

Triangles of the forms $\left(90^{\circ}, 60^{\circ}, 60^{\circ}\right),\left(180^{\circ} / n, 180^{\circ}-180^{\circ} / n, 180^{\circ}-180^{\circ} / n\right)$, $\left(360^{\circ} / n, 90^{\circ}, 90^{\circ}\right)$ for $n$ even, also have non-edge-to-edge tilings in which the tiles of

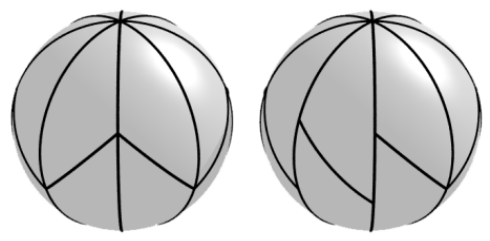

Fig. 8. Edge-to-edge and non-edge-to-edge lune tilings. 

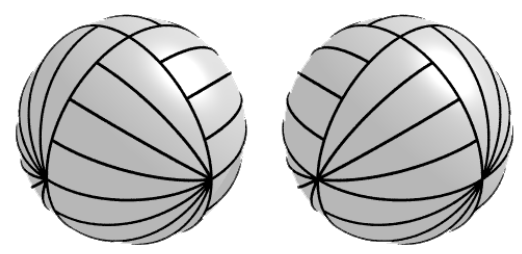

Fig. 9. Tilings with right semilunes.

one or more lunes are rearranged into two congruent convex polygons, one at each pole, and the remaining lunes fill in the supplement of each angle (Fig. 10). We call these swirl tilings; again, the numbers of these tilings are large.

\section{Classification of Tilings: Short Triangles}

Lemma 5. If a short triangle has the vertex vector $(a, b)$, then:

(i) if $a>1$, then $a+b \leq 5$;

(ii) $a \leq 5$.

Proof. If $a+b \geq 5$ and $a \geq 2$ we would have $2 \alpha+4 \beta<a \alpha+b \beta=360^{\circ}$, and hence $\alpha+2 \beta>180^{\circ}$.

We also note that $(2,0)$ and $(1,0)$ yield degenerate triangles, $(1,1)$ yields a reflex triangle with excess greater than $180^{\circ}$ which cannot tile the sphere, and $(1,2)$ yields a triangle with excess $180^{\circ}$, four copies of which tile in an edge-to-edge fashion. Vertex vectors $(0,1)$ and $(0,2)$ are impossible with $\alpha>\beta$, and $(0,3)$ requires $\alpha>\beta=120^{\circ}$. Thus the spherical excess is greater than $180^{\circ}$, making the number of tiles, $n$, at most three. However, this requires $\alpha=360^{\circ}$, and the triangle is degenerate. We may thus restrict our attention to the vertex vectors $(5,0),(4,1),(4,0),(3,2),(3,1),(3,0),(2,3),(2,2),(2,1)$, $(1, b)$ for $b \geq 3$, and $(0, b)$ for $b \geq 4$.

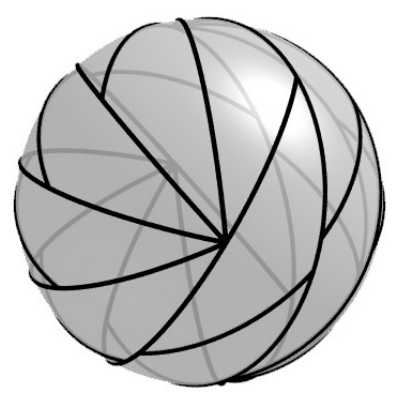

Fig. 10. A swirl tiling. 
Proposition 3. The only short triangle that tiles the sphere and has a $(5,0)$ vertex vector is the $\left(72^{\circ}, 60^{\circ}, 60^{\circ}\right)$ triangle, which only tiles edge-to-edge.

Proof. A triangle with the vertex vector $(5,0)$ must also have at least one other vertex vector. The Angle Comparison Lemma (ACL) rules out every vector with $a+b \leq 5$; consulting Table 1 , we find that the only possibilities are $(1, b), b \geq 5$, and $(0, b), b \geq 6$. If $(5,0)$ and $(1,2 n+1)$ are vertex vectors, they are the only ones, and neither splits; if $(5,0)$ and $(1,2 n)$ are vertex vectors, so is $(3, n)$ which we have already ruled out. Finally, if $(5,0)$ and $(0, b)$ are vertex vectors, the Sphericity Lemma (SL) requires $3(b-4)<8$; so $b=6$. This gives the $\left(72^{\circ}, 60^{\circ}, 60^{\circ}\right)$ triangle. This tiles edge-to-edge [3], [11], but (Proposition 1) does not tile in any other way.

Proposition 4. No short triangle that tiles the sphere has a $(4,1)$ vertex vector.

Proof. First, we note that there are no short triangles that tile edge-to-edge and have this vertex vector [3], [11], [12]. The vector $(4,1)$ rules out any other vector with $a+b \leq 5$; we are thus left only with the options $(1, b), b \geq 5$, and $(0, b), b \geq 6$, for a second vertex vector.

If $(1, b)$ is a vertex vector, the SL implies $2(b-4)<3$, and so $b=5$. The vectors $(4,1)$ and $(1,5)$ are not consistent with any others, and neither splits; so this option is ruled out.

If $(0, b)$ is a vertex vector, the SL implies $2(b-4)<6$, whence $b<7$; so the vector must be $(0,6)$. This splits, but yields no other vertex vectors. The triangle has angles of $\left(75^{\circ}, 60^{\circ}, 60^{\circ}\right)$ and an excess of $15^{\circ}$; its area is thus $1 / 48$ of the sphere.

The large angle of each triangle must belong to a $(4,1)$ vertex, which must have the configuration of vertex $A$ in Fig. 11. The three other triangles at $B$ are forced; it therefore follows that any tiling of the sphere with these triangles can be divided into such eight-element supertiles, disjoint from each other.

However, we shall see that these supertiles cannot tile the sphere. If the reflex angle at $C$ is filled by anything other than the vertex $C^{\prime}$ of another supertile, a reflex angle

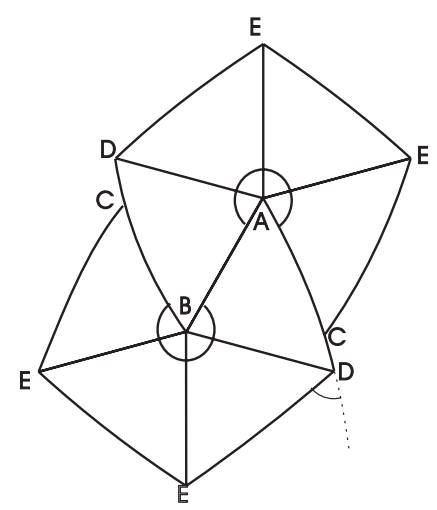

Fig. 11. The $(4,1)$ supertile 


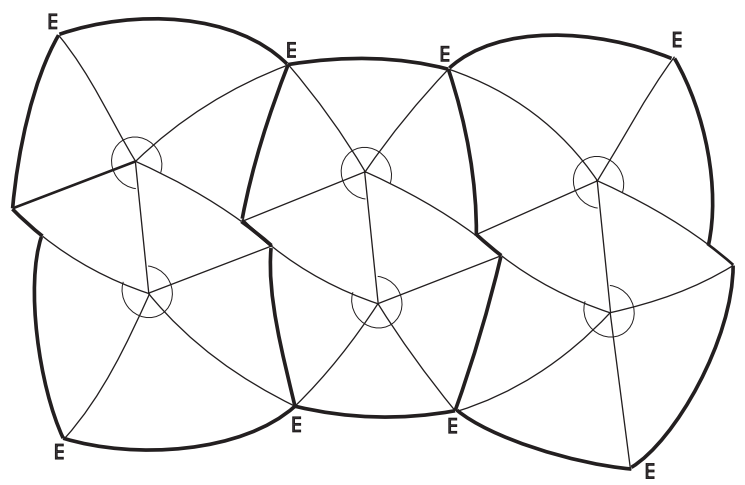

Fig. 12. How supertiles fit together.

of $60^{\circ}$ is created at $D$ that cannot be filled by any vertex of a supertile. However, when supertiles fit together in this way, one edge $\overline{E E}$ is left exposed at the top and bottom (Fig. 12).

These edges form a polygonal path with all edges of length $L$, and all angles $120^{\circ}$; the region bounded by this path cannot be a supertile. We conclude that the vertex vector $(4,1)$ is not compatible with any others. As it clearly cannot be used on its own, it is not used in any tilings.

Note. If two more triangles are added to the top and bottom of a supertile, their apices are antipodal. Moreover, the union of these ten tiles can be considered as a $75^{\circ}$ lune with zigzag edges, which fit together. Four such near-lunes can be fitted together, covering 40/48 of the sphere; and (Fig. 13) six more triangles can be added, leaving only eight exposed edges, two partial. The figure shows the tiling and a " $360^{\circ}$ fisheye" view from inside.

It is interesting to note that in this partial tiling, one tile (shown as the outside of the fisheye view) has three complete layers of tiles around it, though there does not exist a full
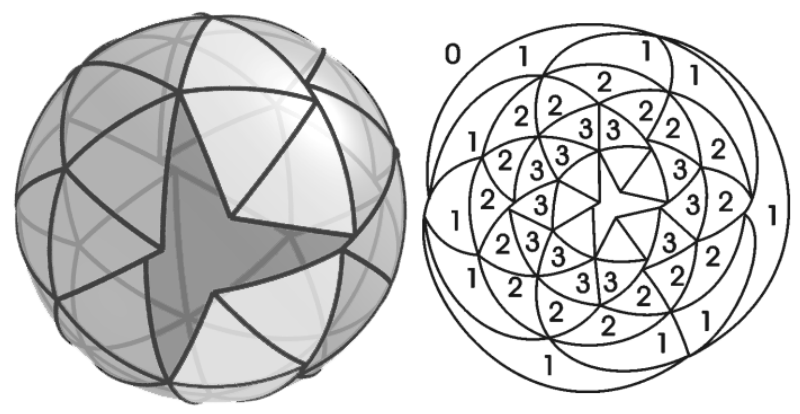

Fig. 13. A near miss. 
tiling; the Heesch number of this tile is thus 3 [6]. In the Euclidean plane, nonconvex tiles with Heesch number 5 are known [7]. Mann conjectures [8] that no convex Euclidean tile has a Heesch number greater than 1.

Alternatively, $240\left(75^{\circ}, 60^{\circ}, 60^{\circ}\right)$ triangles can be formed into 24 zigzag lunes, which yield an exact fivefold tiling of the sphere.

Proposition 5. The only short triangle that tiles the sphere and has a $(4,0)$ vertex vector is the $\left(90^{\circ}, 60^{\circ}, 60^{\circ}\right)$ triangle, which can tile edge-to-edge.

Proof. Such a triangle (i.e., a right triangle) must also have at least one other vertex vector; the ACL rules out $(5,0),(4,1)$, and all vertex vectors with $a+b \leq 4$. In conjunction with $(3,2)$, the resulting triangle is not spherical. With $(2,3)$, we obtain the $\left(90^{\circ}, 60^{\circ}, 60^{\circ}\right)$ triangle.

If $(4,0)$ is the only vertex vector with $a>1$, there cannot be a vector of the form $(0,2 n)$, which would imply the existence also of $(2, n)$. Vectors of the forms $(1, b)$ and $(0,2 n+1)$ do not split; thus, the only split vector would be $(4,0) / 2$. However, then (Lemma 4) any such tiling would be edge-to-edge; and [3], [11] there are no such tilings.

Proposition 6. The triangle $\left(80^{\circ}, 60^{\circ}, 60^{\circ}\right)$ is the only short triangle with a $(3,2)$ vertex vector that tiles the sphere; and it does not do so in an edge-to-edge fashion.

Proof. The ACL and SL rule out all vertex vectors with $a \geq 2$. If a triangle has vertex vectors $(3,2)$ and $(1,2 n+1)$, that triangle has no vertex vector that splits; if $b=2 n$, the triangle also has a vertex vector $(2, n+1)$ and these have already been ruled out. The vertex vector $(0, b)$ is ruled out by the ACL for $b \leq 5$, and by the SL for $b \geq 8$. For $b=7$ neither vector splits, and there are no others. For $b=6$ we get the triangle $\left(80^{\circ}, 60^{\circ}, 60^{\circ}\right)$, which tiles the sphere with 36 tiles (Fig. 14) but does not do so in an edge-to-edge fashion.

In fact, this triangle tiles in three essentially distinct ways; it is shown in [4] that these are the only ways in which it tiles. In Davies' classification [3], it was a particularly refractory case, requiring the construction of a net of 18 triangles before a vertex was found that could not be completed within an edge-to-edge tiling. This may perhaps have

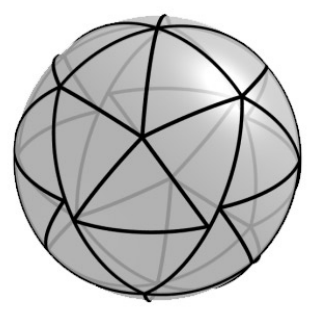

Fig. 14. A tiling with the triangle $\left(80^{\circ}, 60^{\circ}, 60^{\circ}\right)$. 
something to do with the fact that the tiling comes so close to being edge-to-edge, and tiles in multiple ways that are not edge-to-edge.

It is also noteworthy that this triangle has three acute angles; as we see from Table 1, obtuse and right triangles predominate among isosceles triangles that tile the sphere. The only other acute isosceles triangles that tile the sphere are the $\left(72^{\circ}, 60^{\circ}, 60^{\circ}\right)$ triangle and the infinite family $\left((360 / n)^{\circ},\left(90^{-} 90 / n\right)^{\circ},(90-90 / n)^{\circ}\right)$ for $n>4$.

As before, bisection yields a scalene right triangle $\left(90^{\circ}, 60^{\circ}, 40^{\circ}\right)$ that tiles the sphere but not edge-to-edge; there is not any obvious extension to an infinite family of scalene triangles that tile.

Proposition 7. The triangle $\left(100^{\circ}, 60^{\circ}, 60^{\circ}\right)$ is the only short triangle with a $(3,1)$ vertex vector that tiles the sphere; and it does not do so in an edge-to-edge fashion.

Proof. The ACL rules out any other vertex vector with $a \geq 3$ or $a+b \leq 4$, as well as $(2,1)$; so a triangle with $(3,1)$ has no vector of the form $(2, b)$. Nor can it have a vector of the form $(1, b)$; if $b$ is even, there are only two vectors, neither of which splits; and if $b=2 n+1$, then $(2, n+1)$ would also be a vector. We note that [3], [11], [12] none of these triangles without split vectors actually tile the sphere.

If a short triangle with vertex vectors $(3,1)$ and $(0, b)$ is spherical, the SL requires that $b<10$. If the tiling is not edge-to-edge, $b$ must be even; hence we have $b=6$ or $b=8$. In either case, the triangles must form six-triangle supertiles (Fig. 15).

If $b=6$, we have the triangle $\left(100^{\circ}, 60^{\circ}, 60^{\circ}\right)$, and the supertiles fit together uniquely to form a tiling of the sphere (Fig. 16). If, on the other hand, $b=8$, the reflex angles of this supertile have measure $360^{\circ}-3 \beta$ and the gap cannot be filled by the $2 \beta$ angles of the supertile; we conclude that this triangle does not tile the sphere.

Note. Bisecting this triangle yields a scalene right triangle, $\left(90^{\circ}, 60^{\circ}, 50^{\circ}\right)$ that tiles the sphere (with 36 tiles) but does not tile edge-to-edge. Moreover, the $\left(100^{\circ}, 60^{\circ}, 60^{\circ}\right)$ triangle is a member of a countably infinite family of (otherwise scalene) triangles with angles $\left(120^{\circ}-60^{\circ} / n, 60^{\circ}, 180^{\circ} / n\right), n>3$, which tile the sphere in a similar pattern with $n$ supertiles meeting at each pole. (Interestingly, all the tilings in this family are two-colorable!)

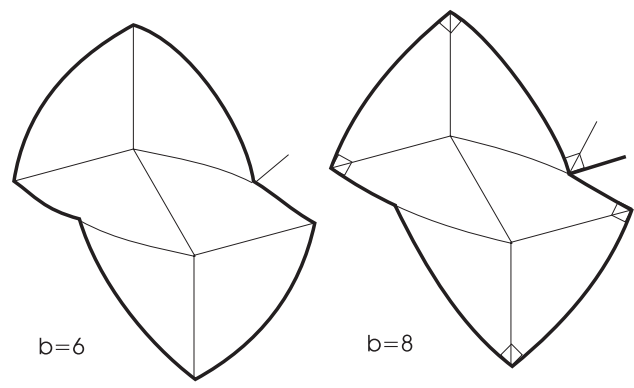

Fig. 15. The supertiles forced for $(3,1),(0, b)$. 


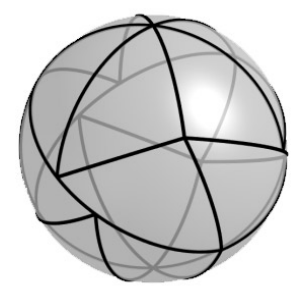

Fig. 16. The tiling with the triangle $\left(100^{\circ}, 60^{\circ}, 60^{\circ}\right)$

Proposition 8. Any short triangle that tiles the sphere and has the vertex vector $(3,0)$ can tile edge-to-edge.

Proof. The vector $(3,0)$ rules out any other vector with $a \geq 3$, and also $(2,1)$. With $(2,2)$, it yields the triangle $\left(120^{\circ}, 60^{\circ}, 60^{\circ}\right)$, which tiles edge-to-edge [3], [11], [12]. The triangle with vertex vectors $(3,0)$ and $(2,3)$ also has $(1,6)$ and $(0,9)$, but none of these split, so it has no non-edge-to-edge tiling (and, in fact, does not tile at all). The vertex vectors $(3,0)$ and $(2,3)$ yield the triangle $\left(120^{\circ}, 30^{\circ}, 30^{\circ}\right)$, which tiles edge-to-edge.

A triangle with vertex vectors $(3,0)$ and $(1,2 n+1)$ has only those vectors, neither of which splits. With vertex vectors $(3,0)$ and $(1,2 n)$, we also have $(2, n)$, so these triangles have already been dealt with.

Finally, if a spherical triangle has vertex vectors $(3,0)$ and $(0, b)$, we must have $b<12$ by the SL. If $b$ is divisible by 3 there are other vertex vectors, already dealt with; if $b=2 n$ we obtain the triangles $\left(120,180^{\circ} / n, 180^{\circ} / n\right), n=2,3,4,5$, all of which tile edge-to-edge; and for $b=5,7,11$ no vector splits and the triangle fails to tile.

Proposition 9. Any short triangle that has the vertex vector $(2,3)$ and tiles the sphere also has some vertex vector with $a>2$.

Proof. Table 1 shows that the only short triangle that tiles edge-to-edge and has the vertex vector $(2,3)$ is the $\left(90^{\circ}, 60^{\circ}, 60^{\circ}\right)$ triangle; and this has the vertex vector $(4,0)$. Any other triangle with $(2,3)$ and tiling the sphere must therefore have a split vertex vector. A triangle with $(2,3)$ and $(1, n)$ also has $(0,2 n-3)$, and none of these split; we can thus restrict our attention to the triangles with $(2,3)$ and $(0,2 n), n>3$. (If $n=2$ the triangle is not short; if $n=3$ we obtain the $\left(90^{\circ}, 60^{\circ}, 60^{\circ}\right)$ triangle.)

If $n>3$, then $\alpha>90^{\circ}$ and there are no other vertex vectors; in particular, the only split vector is $(0,2 n) / 2$. Consider, then, the neighborhood of a $(2,3)$ vertex $A$. However the edges are paired, there must be at least one short edge $\overline{A B}$ paired with a long edge (Fig. 17).

Either vertex $B$ at the other end of the short edge has an angle of measure $\alpha$ and cannot be completed (Fig. 17(a)), or it has the split vector $(0,2 n) / 2$. This split vertex cannot be related in the same way to any other $(2,3)$ vertex; so there must be at least as many $(0,2 n) / 2$ split vertices as there are $(2,3)$ vertices. However, this requires at least $7 / 3$ times as many $\beta$ angles as there are $\alpha$ angles, which is impossible. 

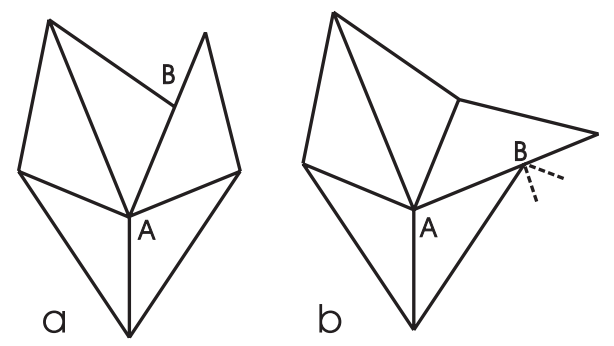

Fig. 17. Typical neighborhoods of a $(3,2)$ vertex $A$.

Proposition 10. Any short triangle that has the vertex vector $(2,2)$ tiles the sphere if and only if it also has another vector, of the form $(0, n)$ for some $n$; and it tiles edge-to-edge if and only if $n$ is even.

Proof. As a tiling of the sphere with short triangles requires twice as many small vertices as large vertices, the triangle must have another vertex vector with $a<2<b$. If it has $(1, b)$ it also has $(0,2 b-2)$; so in any case it has a vertex vector of the form $(0, n)$. It is easily verified that, for any $n, 2 n$ copies of the $\left((180-360 / n)^{\circ},(360 / n)^{\circ},(360 / n)^{\circ}\right)$ triangle tile the sphere; pairs of them form lunes with an angle of $\left.(360 / n)^{\circ}\right)$, and $n$ of these lunes tile the sphere.

These triangles are the isosceles semilunes. They tile in a large number of ways: two examples are shown in Fig. 8.

Proposition 11. If $n$ is odd, the $\left((180-360 / n)^{\circ},(360 / n)^{\circ},(360 / n)^{\circ}\right)$ triangle tiles the sphere in

$$
\frac{1}{4 n}\left\{\sum_{i=1}^{n} 2^{\operatorname{gcd}(n, i)}+n 2^{(n+1) / 2}\right\}
$$

essentially distinct ways.

Proof. As $n$ is odd, the triangle has only the vertex vectors $(2,2)$ and $(0, n)$, and the latter does not split. We have $\varepsilon=(360 / n)^{\circ}$, so there are $2 n$ tiles. Let $p$ be the number of $(2,2)$ vertices plus half the number of $(2,2) / 2$ split vertices, and let $q$ be the number of $(0, n)$ vertices; we have $2 p=n$ large angles and $2 p+n q=2 n$ small angles. Solving, we find that $p=n / 2$ and $q=2$.

Around either of the $(0, n)$ vertices is an irregular rosette of triangles. Each of these triangles has one entire short edge not covered by other triangles of the rosette (Fig. 18). The small angle of the exposed short edge must either be on a partially exposed long edge ( $\overline{A C}$ in the diagram) or at a vertex shared by two small angles as at $E$. Therefore, no edge may overhang that angle, as in one case it would leave an exposed segment of length $L-S$ that could not be covered, and in the other case it would require a split vertex with two or more small angles (impossible for odd $n$ ). We conclude that each exposed short edge must be covered by the short edge of another triangle. Moreover, as 


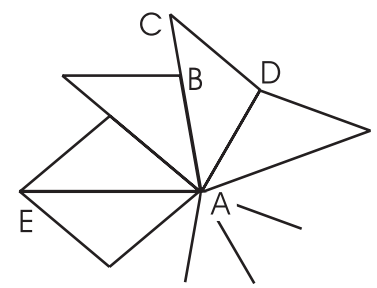

Fig. 18. Typical neighborhood of a $(0, n)$ vertex $A$.

we cannot put a second large angle at the split vertex $B$ or a third at $D$, every triangle of the rosette must form half of a lune.

The sphere is thus divided into $n$ lunes, each subdivided into two triangles (recall Fig. 8). We enumerate the essentially distinct ways of performing this subdivision using Burnside's theorem:

$$
\mid \text { tilings } \mid=\frac{1}{|G|}\left\{\sum_{f \in G}|F i x(f)|\right\} \text {, }
$$

where $G$ is the symmetry group of the lune tiling and, for any element $f$ of $G, F i x(f)$ is the number of subdivisions fixed by $f$.

The symmetry group of the lune tiling consists of $n$ rotations about the axis through its vertices (including the identity), $n$ rotations about axes in the equatorial plane (one through the center of each longitudinal edge), $n$ rotatory reflections, and $n$ reflections. As there are an odd number of lunes, it is not possible for each orbit of an isometry to have even length; therefore, as the action of a reflection on a subdivision is nontrivial, it follows that no reflecting isometry can fix any subdivision. Hence $\mid$ Fix $(f) \mid=0$ for all reflecting isometries.

If $f$ is a proper isometry, it is either a rotation through $(360 i / n)^{\circ}$ about the polar axis, or through $180^{\circ}$ about an axis in the equatorial plane. In the former case, there are $\operatorname{gcd}(i, n)$ orbits in its action on the $n$ lunes; and in the latter case, there is always one singleton orbit and $(n-1) / 2$ two-element orbits. Each orbit may be subdivided in two ways, and (3) follows.

Note. For $n=3,5,7,9,11,13,15, \ldots$, the numbers of distinct tilings are $1,4,9$, $23,63,190,612, \ldots$ This sequence is closely related to the sequence $2,4,8,20,60$, $188, \ldots$, which enumerates binary necklaces of odd length [10].

Note. Calculation for even $n$ is more complicated for two reasons. Firstly, there are reflecting isometries whose orbits on the set of lunes are all of even length, and which therefore can fix subdivisions. Secondly, and more seriously, the vertex vector $(0, n)$ splits, and there is a third vertex vector $(1,(n+2) / 2)$. (When $n=6$, we have the $\left(120^{\circ}, 60^{\circ}, 60^{\circ}\right)$ triangle, which has a fourth vertex vector, namely $(3,0)$.) These give rise to "swirl" tilings such as that shown in Fig. 10. We shall not attempt to enumerate these here. 


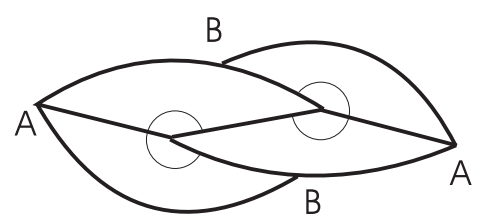

Fig. 19. Supertiles of triangles with vertex vector $(2,1)$.

Proposition 12. The triangle $\left(150^{\circ}, 60^{\circ}, 60^{\circ}\right)$ is the only short triangle with the vertex vector $(2,1)$ that tiles the sphere; and it does not do so edge-to-edge.

Proof. If a triangle has the vertex vector $(2,1)$ it cannot have any other vertex vector with $a \geq 2$; if it has any vector $(1, n)$ it also has $(0,2 n-1)$, and none of these split. As no short triangle with a $(2,1)$ vertex vector tiles the sphere edge-to-edge, these triangles do not tile at all.

We need thus consider only triangles with $(2,1)$ and $(0,2 n)$. These have no other vertex vectors, and necessarily form supertiles as shown in Fig. 19.

When $n=3$, the angle $A$ of the supertile fits the reflex angle $B$, and two supertiles tile the sphere (Fig. 20). For even $n$, $A$ does not divide $B$; for odd $n>3$ two or more angles $A$ are required for each angle $B$, and this is impossible as each supertile has the same number of each.

Note. Bisecting the large angle of the $\left(150^{\circ}, 60^{\circ}, 60^{\circ}\right)$ triangle yields a scalene right triangle that tiles the sphere only in a non-edge-to-edge fashion, with angles $\left(90^{\circ}, 75^{\circ}, 60^{\circ}\right)$.

Note. The $\left(150^{\circ}, 60^{\circ}, 60^{\circ}\right)$ triangle is part of a continuum family of triangles, otherwise scalene, with angles $\left(180^{\circ}-\theta / 2, \theta,\left(180^{\circ}-\theta\right) / 2\right)$ for $\pi / 4<\theta<\pi / 2$.

Finally, we consider the triangles that have no vertex vector $(a, b)$ with $a>2$. As noted above, the vector $(1,2)$ gives a continuous family of triangles, all of which tile the sphere edge-to-edge with four copies; and any other such equation, alone or in combination with other such equations, requires more than twice as many $\beta$ angles as

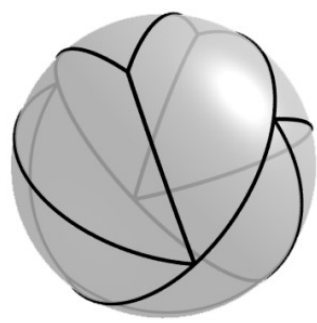

Fig. 20. The tiling with the triangle $\left(150^{\circ}, 60^{\circ}, 60^{\circ}\right)$. 
$\alpha$ angles. We have thus completely classified the short triangles that tile the sphere in a non-edge-to-edge fashion.

\section{Classification of Tilings: Tall Triangles}

In the tall case, many fewer vertex vectors are ruled out by angle-sum considerations. Locally, $(5,0),(4,0),(4,1)$, and all vectors with $a \leq 3, a+b \geq 3$ are realizable. However, while there are non-edge-to-edge tilings with tall triangles, there are no new tiles.

Proposition 13. Any tall triangle that tiles the sphere does so in an edge-to-edge fashion.

Proof. A triangle with the vertex vector $(5,0)$ cannot have $a+b \leq 5$ in any other vector, hence any such vector has $a \leq 3$. A triangle with $(5,0)$ and $(3,2 n+1)$ has only $(1,4 n+2)$ as an alternative vector, and none of these split. Vertex vectors $(5,0)$ and $(3,2 n)$ imply $(4, n)$ which has already been ruled out. Thus $a \leq 2$ in any other vector. However, it is not possible to pair long edges with long edges and short edges with short edges all around a $(5,0)$ vertex, and pairing two edges of different lengths requires a $(1, n)$ split vertex. Thus a tall triangle with $(5,0)$ that tiles the sphere must also have some vertex vector $(2,2 n)$. Now, $(2,2)$ is ruled out by angle inequalities, and $n \geq 3$ yields a nonspherical triangle. This leaves only $(2,4)$ as a potential second vertex vector. These vectors give us the $\left(72^{\circ}, 72^{\circ}, 54^{\circ}\right)$ triangle; but we shall show that this fails to tile.

We say that a $(5,0)$ vertex owns any $(1,2)$ or $(2,4)$ vertex that is joined to it by a short edge, and any $(2,4)$ vertex that is joined to it by a long edge passing through the middle of four $\beta$ angles. Thus, in Fig. 21, $A$ owns $X$ outright, and shares ownership of $Y$ with $B$; but $C$ does not own $Y$. A $(1,2)$ vertex can be owned by at most one $(5,0)$, and a $(2,4)$ by at most two.

Looking at the ways of arranging five $\alpha$ angles around a point, we see that either a $(5,0)$ vertex $A$ owns at least two (in fact, three) $(1,2)$ vertices, or it owns one and has the configuration of Fig. 22(a). In the latter case, we will show that $A$ at least shares a $(2,4)$ vertex.

Suppose $A$ to own neither $B$ nor $C$; it follows that they must be $(5,0)$. If there were a split vertex on the edge $\overline{B D}$, there would be an overhang at $D$, requiring an $\alpha$ corner

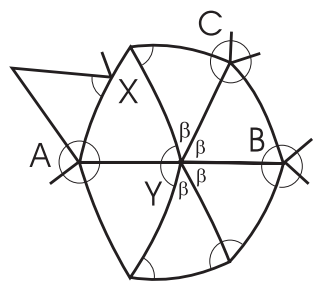

Fig. 21. Ownership of vertices. 


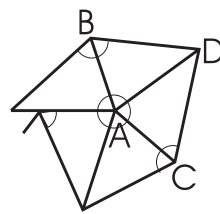

a

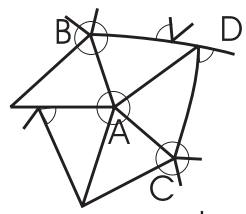

b

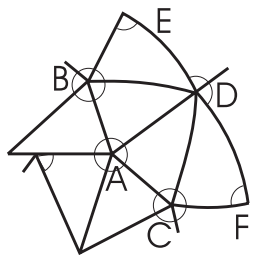

C

Fig. 22. Neighborhood of a $(5,0)$ vertex A that owns only one $(1,2)$ vertex.

to fill it. Then $\overline{C D}$ would be a long edge with an $\alpha$ at each corner, which is impossible (Fig. 22(b)). We therefore conclude that $\overline{B D}$ is a long edge shared by two triangles, $\triangle B D A$ and $\triangle B D E$, and that $\angle B D E=\beta$ (Fig. 22(c)). By the same argument, $\angle C D F$ is as shown and equals $\beta$. We conclude that $D$ is a $(2,4)$ owned at least in part by $A$.

Now, if every $(5,0)$ owns at least two $(1,2)$ vertices outright, or owns one and shares ownership of a $(2,4)$ with at most one other $(5,0)$, it follows that the total number of $\beta$ angles would have to be at least $4 / 7$ times the number of $\alpha$ vertices, which is impossible. Thus no tall triangle has vertex vectors $(5,0)$ and $(2,4)$.

It should be noted that the $\left(72^{\circ}, 72^{\circ}, 54^{\circ}\right)$ triangle, which has $(5,0)$ and $(2,4)$ as vertex vectors, does "locally" tile the sphere, in that it permits a triple cover of the sphere with 20 small vertices meeting at each pole (Fig. 23).

If a triangle has $(4,0)$ as a vertex vector and tiles the sphere, it must also have a second vertex vector $(a, b)$. If $a=0,2$, or 3 it follows immediately that $\beta$ divides $360^{\circ}$; if $a=1$, $\beta$ divides $270^{\circ}$. However, as $\alpha=90^{\circ}$, the area of the triangle is $\beta$, so $\beta$ also divides $720^{\circ}$; it follows that $\beta$ divides $90^{\circ}$. We thus have, in either case, a $\left(90^{\circ}, 90^{\circ}, 360^{\circ} / n\right)$ triangle, discussed in Section 3.

A tall triangle with the vertex vector $(4,1)$ must have some other vertex vector as well. If there were only one other equation with $a=1$ neither would split, and the tiling would be edge-to-edge; but there is [3], [11], [12] no such tiling. Any second vector with $a=3$ or $a=2$ implies a third with $a=0$. As $\beta<\alpha$, we have $b>5$; and for every such $b$ there exists (Section 3 ) an edge-to-edge tiling with vertex equations $(4,1),(0, b)$. These are known to tile edge-to-edge, and (Proposition 2) do not tile in any other way.

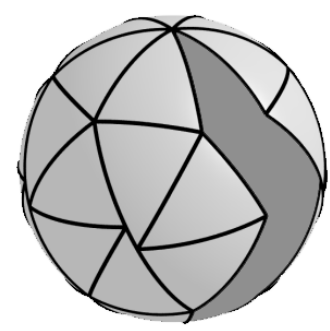

Fig. 23. Three-tenths of the triple cover with $\left(72^{\circ}, 72^{\circ}, 54^{\circ}\right)$ triangles. 
A triangle with vertex vector $(3,0)$ has $\beta>60^{\circ}$ (by Lemma 3) and thus cannot have any vertex vector of the form $(2, b)$. Thus there could be no split vertex including an angle with measure $\alpha$. Thus triangles would have to meet short edge to short edge, in pairs, implying $N$ to be even. However, $\varepsilon>120^{\circ}$, so we have $N<6$ (and thus $N=4$ ), which requires $\beta=120^{\circ}=\alpha$. The triangle is equilateral, and forms the faces of a spherical tetrahedron. It does not tile non-edge-to-edge.

Next, the vector $(3,1)$ implies that $\alpha>90^{\circ}$, and thus that any vector with $a<3$ will have $b>a$. Any such tiling would therefore have to contain at least one $(3,1)$ vertex. Such a vertex would have three short edges and five long edges, and thus a short edge paired with a long edge. This would imply a split vertex joined to the $(3,1)$ by a short edge; as above, we say that the $(3,1)$ owns the split vertex; and it is easily verified that no split vertex is owned by more than one $(3,1)$.

Now, $(3,1)$ rules out $(2,2)$ as a vertex vector, so any split vertex would have the split vector $(2,2 b) / 2, b>1$. If $(3,1)$ owns $n$ vertices, they have between them $3+n$ angles with measure $\alpha$ and $1+2 n$ with measure $\beta$. As $(3+n)<2(1+2 n)$ for $n>0$, and as any other vertices that exist have $a<2 b$, no combination of such vertices will give $\sum a=2 \sum b$ as required.

Finally, any vector with $a=3, b \geq 2, a=2, b \geq 2$, or $a=1$ fails to satisfy $a \geq 2 b$, and forces any other vertex vector with smaller $a$ to do the same. Again, we cannot obtain $\sum a=2 \sum b$ by combining such vertices. This leaves only $(2,1)$; triangles with this vector belong to the same continuous family as the short triangles with $(1,2)$, and the same argument shows that they tile only edge-to-edge.

\section{Conclusion}

We have seen that several of the isosceles spherical triangles which tile the sphere edge-to-edge can also tile it in a non-edge-to-edge fashion, while others can only tile edge-to-edge. We have also exhibited three "sporadic" cases, and one (obvious) infinite family, of spherical triangles that can only tile in a non-edge-to-edge fashion.

This is a first step towards the eventual classification of all triangles that tile the sphere. In future work it will be possible to assume that any as yet undiscovered triangle that tiles the sphere is scalene. Moreover, three new scalene tiles have been discovered, the right triangles obtained by bisection of the triangles of Figs. 14, 16, and 20; and two infinite families of tiles, of which the triangles of Figs. 16 and 20 are special cases.

Two interesting open questions are suggested by this work. Firstly, one may ask whether any spherical tile has a Heesch number exceeding 3. Figure 13 shows that the $\left(75^{\circ}, 60^{\circ}, 60^{\circ}\right)$ triangle comes tantalizingly close to a Heesch number of 4 , but apparently does not achieve it - though a better partial tiling cannot be ruled out.

Secondly, we have seen some examples of multiple covers of the sphere with isosceles triangles. There are several other easy examples, such as all semilunes whose lune angles are rational multiples of $\pi$. This class of examples shows, incidentally, that we may achieve an $n$-tuple cover for any $n$. Schwarz [9] (see also Chapter 6 of [2]) has classified the triangles that yield finite-density multiple covers of the sphere by reflection; they consist of the right semilunes with rational lune angle, and a finite number of others. It 
would be interesting — but probably very difficult — to classify all finite-density tilings, or even just the finite-density edge-to-edge tilings.

\section{Acknowledgements}

I would like to acknowledge the anonymous referee's contributions to the paper, and, in particular, the reference to the paper of Ueno and Agaoka.

\section{References}

1. Azavedo Breda, A.M.R., A class of tilings of $S^{2}$, Geom. Dedicata 44 (1992), 241-253.

2. Coxeter, H.S.M., Regular Polytopes, Dover, New York, 1973.

3. Davies, H.L., Packings of spherical triangles and tetrahedra, Proc. Colloquium on Convexity, Copenhagen, 1965, pp. 42-51.

4. Dawson, R.J.M., An isosceles triangle that tiles the sphere in exactly three ways, Discrete Comput. Geom., this issue, pp. 459-466.

5. Grünbaum, B., and Shepherd, G.C., Tilings and Patterns, Freeman, New York, 1987.

6. Heesch, H., Eine Betrachtung der 11 homogenen Ebenenteilungen, Mathematikunterricht (1968), 66-78.

7. Mann, C., An infinite family of prototiles with Heesch number 5, unpublished.

8. Mann, C., Private communication.

9. Schwarz, H.A., Zur Theorie der hypergeometrischen Reihe, J. Angew. Math. 75 (1873), 292-335.

10. Sloane, N.J.A., and Plouffe, S., The Encyclopedia of Integer Sequences, Academic Press, San Diego, CA, 1995.

11. Sommerville, D.M.Y., Division of space by congruent triangles and tetrahedra, Proc. Roy. Soc. Edinburgh 43 (1923), 85-116.

12. Ueno, Y., and Agaoka, Y., Classification of the Tilings of the 2-Dimensional Sphere by Congruent Triangles, Technical Report 85, Division of Mathematical and Information Sciences, Hiroshima University, 2001.

Received April 29, 2001, and in revised form November 26, 2002. Online publication August 11, 2003. 